\title{
Distributed Multi-radio Channel Allocation in Multi-Hop Ad Hoc Networks
}

\author{
Lin Gao, Xinbing Wang, Youyun Xu, Wen Chen \\ Dept of Electric Engineering \\ Shanghai Jiao Tong University \\ Email: \{gaull,xwang8,xuyouyun, wenchen\}@sjtu.edu.cn
}

\begin{abstract}
Channel allocation was extensively researched in the framework of cellular networks, but it was rarely studied in the ad-hoc wireless networks, especially in the multi-hop ad-hoc networks. In this paper, we study the problem of competitive multi-radio multi-channel allocation in multi-hop wireless networks in detail. We model the channel allocation problem as a static cooperative game, and then derive a min-max coalitionproof Nash equilibrium (MMCPNE) in this game. We study the existence of MMCPNE in the static game and prove the necessary and sufficient conditions for MMCPNE. Finally, we propose a two-step distributed algorithm that enable the selfish players to converge to MMCPNE. ${ }^{1}$
\end{abstract}

\section{INTRODUCTION}

Wireless communication networks are often assigned a certain range of communication medium (e.g., frequency band). Usually this medium is shared by multiple users. Frequency Division Multiple Access (FDMA), which enables more than one user to share a given frequency band, is one of the extensively adopted techniques in wireless networks [17], [19]. In FDMA, the total available bandwidth is divided permanently into a number of distinct sub-bands which is named as channels. Commonly, we refer to the assignment of radio transceivers to these channels as the channel allocation problem. It is undoubted that an efficient channel allocation is essential for the design of wireless networks.

In this paper, we present a game-theoretic analysis of fixed channel allocation strategies of devices that use multiple radios in the multi-hop wireless networks. Static non-cooperative game is a novel approach to solve the channel allocation problem in single-hop networks and Nash equilibrium provides an efficient criterion to evaluate a given channel allocation (e.g., in [11]). However, non-cooperative game does not work in multi-hop networks for the reasons mentioned in Section IV. Hence, we introduce static cooperative game with perfect information into our model. We analyze the scenario of a single collision domain, i.e., where each of the devices can interfere with a transmission of any other device. We first derive a min-max coalition-proof Nash equilibria (MMCPNE) in this game, in which each player would like to maximize its end-to-end rate. Then we study the existence of MMCPNE in this game. Our main result, Theorem 2, shows the necessary condition for the existence of MMCPNE. Furthermore, we propose a two-step algorithm, denoted by MMCP algorithm,

\footnotetext{
${ }^{1}$ This work is supported by NSF China (No. 60702046).
}

which enables the selfish players to converge to MMCPNE from an arbitrary initial configuration. Finally, we present the simulation results of MMCP algorithm from which we conclude that MMCPNE outperforms NE channel allocation scheme in terms of actual data rate of communication link in the multi-hop networks.

The paper is organized as follows. In Section II, we present related work on channel allocation and channel access in wireless networks. In Section III, we introduce our system model which contains multi-hop link. In Section IV, we introduce game-theoretic description of competitive channel allocation in multi-hop networks. In Section V, we provide a comprehensive analysis of the coalition-proof Nash equilibria in the channel allocation game. Additionally, we propose an algorithm to reach the expected MMCPNE and we study the convergence properties of the algorithm in Section VI. Finally, we conclude in Section VII.

\section{RELATED WORK}

There has been a considerable amount of research on channel allocation in wireless networks, especially in cellular networks. Three major categories of channel allocation schemes are always used in cellular networks: fixed channel allocation [1], dynamic channel allocation [2] and hybrid channel allocation [3], which is a combination of both FCA and DCA techniques.

Recently, channel allocation problem is becoming a focus of research again due to the appearance of new communication technologies, e.g., wireless local area networks (WLANs) and wireless mesh networks (WMNs) etc. Using weighted graph coloring method, Mishra et al. propose a channel allocation method for wireless local area networks in [4]. In wireless mesh networks (e.g., as present in [5] and [6]), many researchers have considered devices using multiple radios. Equipping multiple radios in the devices in WMNs, especially the devices acting as wireless routers, can improve the capacity by transmitting over multiple radios simultaneously using orthogonal channels. In the multi-radio communication context, channel allocation and access are also considered as the vital topics.

In all the cited work, the authors make the assumption that the radio devices cooperate with the purpose of the achievement of high system performance. However, this assumption might not hold because the users of these devices are usually 
selfish who would like to maximize their own performance without considering the other users' objective. Game theory provides a straightforward tool to study channel allocation problems in competitive wireless networks. As far as know, game theory has been applied to the CSMA/CA protocol [7], [8] and to the Aloha protocol [9]. Furthermore, on the basis of graph coloring, Halldorsson et al. use game theory to solve a fixed channel allocation problem in [10]. Unfortunately, their model does not apply to multi-radio devices. In wireless adhoc networks (WANETs), Felegyhazi et al. present a gametheoretic analysis of fixed channel allocation strategies of devices that use multiple radios in [11]. The idea is also suitable for wireless sensor network [15], [18]. However, their results can be only applied to single-hop wireless networks without the consideration of multi-hop wireless networks.

\section{SYSTEM MODEL}

We assume that the available frequency band is divided into orthogonal channels of the same bandwidth using the FDMA method (e.g., 8 orthogonal channels in case of the IEEE 802.11a protocol). We denote the set of available orthogonal channels by $\mathcal{C}=\left\{c_{1}, c_{2}, \ldots, c_{N}\right\}$.

In our model, we assume each user participates in only one communication session. Hence we can divide all users into some disjoint groups, denoted by $g_{i, i=1,2, \ldots, L}$, according to different communication sessions. We denote the set of groups by $\mathcal{G}=\left\{g_{1}, g_{2}, \ldots, g_{L}\right\}$. Each group $g_{i}$ corresponds to a communication session $l_{i}$ in wireless networks, hence we denote the set of communication sessions by $\mathcal{L}=\left\{l_{1}, l_{2}, \ldots, l_{L}\right\}$ and $l_{i} \rightleftharpoons g_{i}$, where " $\rightleftharpoons$ " denotes a bidirectional mapping. Additionally, we denote the set of senders in all groups by $\mathcal{U}$ and the set of relaying users by $\mathcal{R}$. Figure 1 presents an example with three communication sessions, where $L=3, \mathcal{L}=\left\{l_{1}, l_{2}, l_{3}\right\}, \mathcal{G}=$ $\left\{\left\{s_{1}, d_{1}\right\},\left\{s_{2}, r_{2_{1}}, d_{2}\right\},\left\{s_{3}, d_{3}\right\}\right\}, \mathcal{U}=\left\{s_{1}, s_{2}, s_{3}\right\}$ and $\mathcal{R}=$ $\left\{r_{2_{1}}\right\}$.

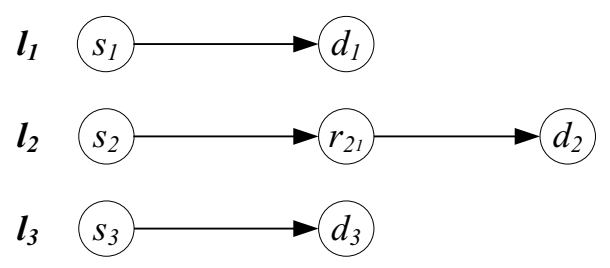

Fig. 1. An example of three communication sessions

We assume each user owns a device equipped with two independent sets of radio transceivers, denoted by $T_{s}$ and $R_{s}$, which used to originate and recept the data packet respectively. Each transceivers set contains $k<|\mathcal{C}|$ radio transmitters, all having the same communication capabilities. The communication between two devices is bidirectional and they always have some packets to exchange. Due to the bidirectional links, the originator and receptor are able to coordinate and thus to select the same channels to communicate. Thus we define the players set as the summation of senders set and relays set, denoted by $\mathcal{D}=\mathcal{U} \cup \mathcal{R}$.
We assume that there is a finite number of players. We further assume that each device can hear the transmissions of any other device if they are using the same channel. This means that the players reside in a single collision domain. Note however that the device cannot communicate directly with all devices except its neighboring devices (e.g., the device equipped by the next-hop player or the previous-hop player) although it can hear the transmission of any other devices.

We denote the number of radios of player $u_{i}$ using channel $c$ by $k_{u_{i}, c}$ for every $c \in \mathcal{C}$. For the sake of suppressing coradios interference in device, we assume that two radios of one device cannot use the same channel, i.e., $k_{u_{i}, c} \leq 1$ for arbitrary users and channels.

We formulate the channel allocation problem with a single stage game, which corresponds to a fixed channel allocation among the players. Each player's strategy consists in defining the number of radios on each of the channels. Hence, we define the strategy of player $u_{i}$ as its channel allocation vector:

$$
x_{u_{i}}=\left(k_{u_{i}, 1}, k_{u_{i}, 2}, \ldots, k_{u_{i},|\mathcal{C}|}\right)
$$

The strategy matrix, denoted by $\mathbb{X}$, is defined by all players' strategy vector:

$$
\mathbb{X}=\left(x_{s_{1}}^{T}, . ., x_{s_{|\mathcal{U}|}^{T}}^{T}, \quad x_{r_{1}}^{T}, . ., x_{r_{2}}^{T}, . ., x_{r_{|\mathcal{R}|} \mid}^{T}, . .\right)^{T}
$$

Furthermore, we denote the strategy matrix except for the strategy of player $u_{i}$ by $\mathbb{X}_{-u_{i}}$ and the strategy matrix of players set $U_{x} \subset \mathcal{D}$ by $\mathbb{X}_{U_{x}}$

Figure 2 presents an example channel allocation strategy in the system of Figure 1 with four available channels $(|\mathcal{C}|=$ 4) and each user device equipped by two radios sets which contains three radios transceivers $(k=3)$ respectively. The tubers at the left of node denote the radios of $R_{s}$ and the remainder tubers denote the radios of $T_{s}$. The number on each radio link denotes the channel used by this radio transceivers.

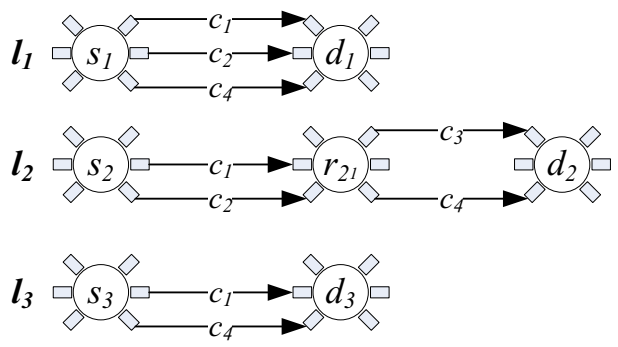

Fig. 2. An example of channel allocation, where $|\mathcal{C}|=4,|\mathcal{U}|=3,|\mathcal{R}|=1$ and $k=3$.

The total number of channels used by player $u_{i}$ can be written as $k_{u_{i}}=\sum_{c} k_{u_{i}, c}$ and it is obviously that $k_{u_{i}} \leq k$. Similarly, the total number of radios using a particular channel $c$ can be written as $k_{c}=\sum_{u_{i}} k_{u_{i}, c}$. In Figure $2, k_{s_{1}}=3$, $k_{s_{2}}=k_{s_{3}}=k_{r_{21}}=2, k_{c_{1}}=k_{c_{4}}=3, k_{c_{2}}=2$ and $k_{c_{3}}=1$.

We denote the total available rate on channel $c$ (i.e., the sum of the achieved bit rate of all players on channel $c$ ) by $R_{c}\left(k_{c}\right)$. We assume that $R_{c}\left(k_{c}\right)$ is independent of the number of radios $k_{c}$ deployed on this channel. We further assume that the total 
available rate $R_{c}$ is shared equally among the radios using that channel. We denote $R_{u_{i}, c}$ as the available rate occupied by player $u_{i}$ on channel $c$ and we can write $R_{u_{i}, c}$ as following:

$$
R_{u_{i}, c}=\frac{k_{u_{i}, c}}{k_{c}} \cdot R_{c}, \quad \forall u_{i} \in \mathcal{D}, c \in \mathcal{C}
$$

We define the one-hop rate of player $u_{i}$ as the total available rate occupied by $u_{i}$ and we denote it by $R_{u_{i}}^{i}$. We can write $R_{u_{i}}^{i}$ as follows.

$$
R_{u_{i}}^{i}=\sum_{c \in \mathcal{C}} R_{u_{i}, c}, \quad \forall u_{i} \in \mathcal{D}
$$

In multi-hop networks, however, the one-hop rate cannot reflect the actual data rate of multi-hop links. Thus we define the end-to-end rate of a communication link as the minimal one-hop rate of all players in the session and we denote it by $R_{l_{i}}^{e}$. We can write $R_{l_{i}}^{e}$ as follows.

$$
R_{l_{i}}^{e}=\min _{u_{i} \in g_{i}} R_{u_{i}}^{i}, \quad \forall l_{i} \in \mathcal{L}
$$

where $u_{i}$ is arbitrary player in group $g_{i}$, i.e., in link $l_{i}$. For single-hop communication link, $R_{l_{i}}^{e}=R_{u_{i}}^{i}$ since there is only one user $u_{i}$ in the link.

We define the payoff of player $u_{i}$ as the achieved data rate in the strategy matrix $\mathbb{X}$, denoted by $P_{u_{i}}(\mathbb{X})$. We refer to each user as a rational player. We also refer to each user as a selfish player, whose objective is to maximize its own payoff in the network. Thus we can formulate the multi-radio channel allocation problem as a static game.

\section{NASH EQUILIBRIA}

In single-hop networks, the multi-radio channel allocation problem can be formulated as a non-cooperative game (e.g., in [11]) with one-hop rate as the payoff function, i.e., $P_{u_{i}}(\mathbb{X})=$ $R_{u_{i}}^{i}, \forall u_{i} \in \mathcal{D}$.

In order to study the strategic interaction of the players in static non-cooperative game, we first introduce the concepts of Nash equilibrium [14], [20].

Definition 1: (Nash Equilibrium - NE): The strategy matrix $\mathbb{X}^{*}=\left\{x_{u_{1}}^{*}, \ldots, x_{u_{|\mathcal{D}|}^{*}}^{*}\right\}$ defines a Nash Equilibrium (NE), if for every player $u_{i}$, we have:

$$
P_{u_{i}}\left(x_{u_{i}}^{*}, \mathbb{X}_{-u_{i}}^{*}\right) \geq P_{u_{i}}\left(x_{u_{i}}^{\prime}, \mathbb{X}_{-u_{i}}^{*}\right)
$$

for every strategy $x_{u_{i}}^{\prime}$.

The definition of NE expresses the resistance to the deviation of a single player in non-cooperative game. In other words, in a NE none of the players can unilaterally change its strategy to increase its payoff. However, non-cooperative game is not suitable for the system in which some players may jointly change their strategies to increase their payoff, e.g., multi-hop networks. Thus we formulate the problem as a cooperative game in multi-hop networks.

In cooperative game, it might be possible that some players collude to increase their payoff at the expense of other players. Such a collusion is called a coalition. We denote the set of all coalitions by $\mathcal{Q}$. We can generalize the notion of NE for coalitions as defined in [16].

Definition 2: (Coalition-Proof Nash Equilibrium - CPNE): The strategy matrix $\mathbb{X}^{c p}$ defines a coalition-proof Nash Equilibrium, if for every coalition $\mathrm{Co}_{i}$, we have:

$$
P_{u_{i}}\left(\mathbb{X}_{c o_{i}}^{c p}, \mathbb{X}_{-c o_{i}}^{c p}\right) \geq P_{u_{i}}\left(\mathbb{X}_{c o_{i}}^{\prime}, \mathbb{X}_{-c o_{i}}^{c p}\right), \quad \forall u_{i} \in c o_{i}
$$

for every strategy set $\mathbb{X}_{c o_{i}}^{\prime}$.

This means that no coalition can deviate from $\mathbb{X}^{c p}$ such that the payoff of at least one of its members increases and the payoff of other members do not change.

In our model, we define coalition as the players belonging to the same communication link, e.g., $s_{2}$ and $n_{2_{1}}$ in Figure 1 . We denote the coalition by $\mathrm{co}_{i}$ and $\mathcal{Q}=\left\{\mathrm{co}_{1}, \mathrm{co}_{2}, \ldots, \mathrm{co}_{L}\right\}$. In Fig. $1, c o_{1}=\left\{s_{1}\right\}, c o_{2}=\left\{s_{2}, r_{2_{1}}\right\}$ and $c o_{3}=\left\{s_{3}\right\}$. Recall the definition of CPNE, we find that CPNE is not strictly suitable for multi-hop networks as we define the coalition as above. In detail, CPNE define such a situation that each player in the coalition cannot improve its payoff without decreasing other players payoff. It might be possible that any player in the coalition achieve a low payoff in the CPNE. Figure 3 presents an example of CPNE where $|\mathcal{C}|=6, k=3$, player $u_{1}, u_{2}$ and $u_{3}$ formulate a coalition and their payoff $P_{u_{1}} \ll P_{u_{2}}=P_{u_{3}}$. As we mentioned above, player $u_{1}, u_{2}$ and $u_{3}$ belong to the same communication link, and thus the actual data rate of $u_{2}$ and $u_{3}$ is the minimal one-hop rate of $u_{1}, u_{2}$ and $u_{3}$, i.e., 0.75 , which is much lower than the payoff of themselves.

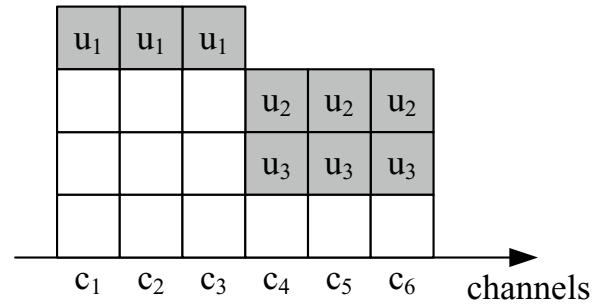

Fig. 3. An example of CPNE channel allocation, where $u_{1}, u_{2}$ and $u_{3}$ formulate a coalition.

Hence, we define a min-max coalition-proof Nash equilibrium (MMCPNE) in the cooperative game, in which players make their decisions so as to improve the minimal rate of players in the coalition. We generalize the notion of MMCPNE as following:

Definition 3: (Min-Max Coalition-Proof Nash Equilibrium - MMCPNE): The strategy matrix $\mathbb{X}^{m m}$ defines a special coalition-proof Nash Equilibrium, if for every coalition $\mathrm{Co}_{i}$, we have:

$$
\min _{u_{i} \in c o_{i}} R_{u_{i}}^{i}\left(\mathbb{X}_{c o_{i}}^{m m}, \mathbb{X}_{-c o_{i}}^{m m}\right) \geq \min _{u_{i} \in c o_{i}} R_{u_{i}}^{i}\left(\mathbb{X}_{c o_{i}}^{\prime}, \mathbb{X}_{-c o_{i}}^{m m}\right)
$$

for every strategy set $\mathbb{X}_{c o_{i}}^{\prime}$.

From definition 3, we can easily find that the payoff function of MMCPNE is exactly the end-to-end rate, i.e., $P_{u_{i}}(\mathbb{X})=$ $R_{l_{i}}^{e}$. Recall the example in Figure 3, the actual data rate of $u_{1}, u_{2}$ and $u_{3}$ increases to 0.917 , if we do $\left(u_{1}, c_{1}\right) \leftrightarrows\left(u_{2}, c_{4}\right)$ 
and $\left(u_{1}, c_{2}\right) \leftrightarrows\left(u_{3}, c_{5}\right)$ at the same time, where $\left(u_{i}, c_{m}\right) \leftrightarrows$ $\left(u_{j}, c_{n}\right)$ means exchanging the radio of $u_{i}$ in channel $c_{m}$ and the radio of $u_{j}$ in channel $c_{n}$.

\section{EXISTENCE OF MMCPNE}

In this section, we study the existence of Nash equilibria and MMCPNE in the single collision domain channel allocation game.

In our model, we assume that $|\mathcal{D}| \cdot k>|\mathcal{C}|$, hence the devices have a conflict during the channel allocation process. We retrospect the work done by Mark Felegyhazi in [11]. The authors study in detail the problem of competitive multi-radio multi-channel allocation in single-hop wireless networks, i.e., $\mathcal{R}=\emptyset$ and $\mathcal{D}=\mathcal{U}$, and propose the conditions for Nash equilibria as the following theorem ${ }^{2}$.

Theorem 1: Assume that $|\mathcal{U}| \cdot k>|\mathcal{C}|$. Then a channel allocation $\mathbb{X}^{*}$ is a NE iff the following conditions hold:

- $k_{u_{i}, c} \leq 1$ and $k_{u_{i}}=k$ for any $u_{i} \in \mathcal{U}, c \in \mathcal{C}$ and

- $\delta_{b, c} \leq 1$ for any $b, c \in \mathcal{C}$

where $\delta_{b, c}=k_{b}-k_{c}$ denotes the difference of radios number between channel $b$ and $c$.

Note however, that this theorem does not work in multihop wireless networks, as shown in Figure 3. We first derive a new single-hop model from the original multi-hop model, denoted by concomitant model, as we consider all multi-hop links as multiple independent single-hop links. In this paper, we refer to NE (or Nash equilibrium) as any channel allocation strategies which satisfy the conditions in theorem 1, i.e., any Nash equilibrium in the concomitant model.

In the following, we study the existence of MMCPNE for coalition set $\mathcal{Q}$ in multi-hop networks. For simplicity, we assume that any communication session contains at most 2 hops, i.e., any coalition $\mathrm{Co}_{i}$ contains at most 2 players. Note however, that it can be easily extended to the system in which any coalition contains more than 2 players.

Although we cannot guarantee every MMCPNE strategy is NE strategy of the concomitant model, we find that most of the MMCPNE strategies hold Nash equilibrium through repetitious experiments. We define the coalition efficiency of any coalition $\mathrm{CO}_{i}$ as the ratio of the achieved payoff of $\mathrm{CO}_{i}$ to the average rate per user. From the results of experiments, we find that the MMCPNE strategies which belongs to the set of NE strategies of the concomitant model always show high coalition efficiency. We show this property as the following proposition.

Proposition 1: Assume that there exists a coalition efficient MMCPNE channel allocation $\mathbb{X}$, then $\mathbb{X}$ holds Nash equilibrium in the concomitant model, i.e., the conditions of theorem 1 hold.

For the remainder of the paper, we focus on the MMCPNE strategy within the set of NE strategy of the concomitant model, i.e., the coalition efficient MMCPNE.

\footnotetext{
${ }^{2}$ Note that we omit the second type of Nash equilibria proposed by Mark Felegyhazi, in which some players use multiple radios in some channels.
}

Players in a coalition can help each other in two ways. The first possibility is a player relocates its radios to improve the rate of its confederate when two players share any channels. This property is expressed as the following lemma.

Lemma 2: Assume that there exists a coalition $\mathrm{co}_{i}=$ $\left\{u_{1}, u_{2}\right\}$ and $R_{u_{1}}^{i} \neq R_{u_{2}}^{i}$ in a NE channel allocation $\mathbb{X}$. If there exists two channels $c_{1} \in \mathcal{C}^{+}$and $c_{2} \in \mathcal{C}^{-}$such that $k_{u_{1}, c_{1}}=1$ and $k_{u_{2}, c_{1}}=1$ whereas $k_{u_{1}, c_{2}}=0$ and $k_{u_{2}, c_{2}}=0$, then $\mathbb{X}$ is not MMCPNE.

In Lemma 2 , we denote $\mathcal{C}^{+}$as the set of channels with the maximum number of radios, i.e., where any $b \in \mathcal{C}^{+}$has $k_{b}=\max _{c \in \mathcal{C}} k_{c}$. Similarly, we denote the set of the remaining channels by $\mathcal{C}^{-}$. We denote the number of radios of any channel in $\mathcal{C}^{+}$and $\mathcal{C}^{-}$by $\delta^{+}$and $\delta^{-}$respectively.

An example of any NE channel allocation corresponding to Lemma 2 is shown in Figure 4, where $|\mathcal{C}|=6, k=4$ and $u_{1}, u_{2}$ formulate a coalition. According to Lemma 2, it cannot be a MMCPNE, since we can increase the payoff of $u_{1}$ and $u_{2}$ by moving $u_{1}$ from channel $c_{4}$ to $c_{6}$.

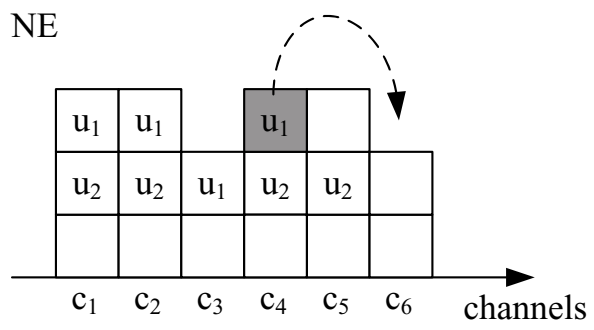

Fig. 4. An example of a NE channel allocation corresponding to Lemma 2.

In some cases the assumption of unequal rates of two players, i.e., $R_{u_{1}}^{i} \neq R_{u_{2}}^{i}$, might not hold. In other word, it is possible that $R_{u_{1}}^{i}=R_{u_{2}}^{i}$. In such cases, the Lemma 2 may no longer hold. Thus we show another necessary condition as follows.

Lemma 3: If there exists a coalition $\mathrm{co}_{i}=\left\{u_{1}, u_{2}\right\}$ and multiple channels $\left\{x_{1}, x_{2}, \ldots\right\} \in \mathcal{C}^{+}$and $\left\{y_{1}, y_{2}, \ldots\right\} \in \mathcal{C}^{-}$ such that $k_{u_{1}, x_{i}}=1$ and $k_{u_{2}, x_{i}}=1, \forall i$ whereas $k_{u_{1}, y_{j}}=0$ and $k_{u_{2}, y_{j}}=0, \forall j$, then NE channel allocation $\mathbb{X}$ is not MMCPNE.

The players in a coalition can also improve their payoff if they mutually exchange some radios of each other. We show this necessary conditions as the following lemma.

Lemma 4: Assume that there exists a coalition $\mathrm{co}_{i}=$ $\left\{u_{1}, u_{2}\right\}$ and $R_{u_{2}}^{i}-R_{u_{1}}^{i}>\left(1 / \delta^{-}-1 / \delta^{+}\right)$in a NE channel allocation $\mathbb{X}$. If there exists two channels $c_{1} \in \mathcal{C}^{+}$and $c_{2} \in \mathcal{C}^{-}$ such that $k_{u_{1}, c_{1}}=1$ and $k_{u_{2}, c_{1}}=0$ whereas $k_{u_{1}, c_{2}}=0$ and $k_{u_{2}, c_{2}}=1$, then $\mathbb{X}$ is not MMCPNE.

We show an example of any NE channel allocation corresponding to Lemma 4 in Figure 5, where $|\mathcal{C}|=6, k=3$ and $u_{1}, u_{2}$ formulate a coalition. According to Lemma 4, it cannot be a MMCPNE, since we can increase the payoff of $u_{1}$ and $u_{2}$ by exchange their radios in channel $c_{3}$ and $c_{5}$.

We divide the radios of any player $u_{i}$ in a NE channel allocation $\mathbb{X}^{*}$ into two sets. We define the set of radios $r_{u_{i}}^{+}$with 


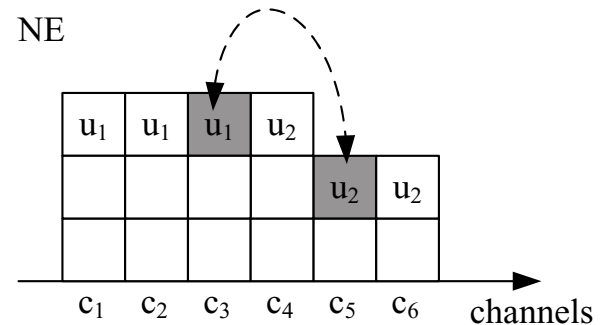

Fig. 5. An example of a NE channel allocation corresponding to Lemma 4.

the radios in $\mathcal{C}^{+}$. Similarly, we define the set of radios $r_{u_{i}}^{-}$with the radios in $\mathcal{C}^{+}$. We denote the size of two sets by $k_{u_{i}}^{+}$and $k_{u_{i}}^{-}$ respectively, i.e., $k_{u_{i}}^{+}=\left|r_{u_{i}}^{+}\right|$and $k_{u_{i}}^{-}=\left|r_{u_{i}}^{-}\right|$. It is easy to see that $k_{u_{i}}^{+}+k_{u_{i}}^{-}=k$. In Figure $4, k_{u_{1}}^{+}=3, k_{u_{1}}^{-}=1, k_{u_{2}}^{+}=4$ and $k_{u_{2}}^{-}=0$. In Figure $5, k_{u_{1}}^{+}=3, k_{u_{1}}^{-}=0, k_{u_{2}}^{+}=1$ and $k_{u_{2}}^{-}=2$. Now we can extend Lemma 4 to a more general situation.

Lemma 5: Assume that there exists a coalition $\mathrm{CO}_{i}=$ $\left\{u_{1}, u_{2}\right\}$ and $\left|k_{u_{1}}^{+}-k_{u_{2}}^{+}\right|>1$ in a NE channel allocation $\mathbb{X}$, then $\mathbb{X}$ is not MMCPNE.

Based on the previous lemmas, we prove the necessary conditions that enables a given NE allocation to be MMCPNE and we present it as the following theorem.

Theorem 2: Assume that there exists a coalition $c o_{i}=$ $\left\{u_{1}, u_{2}\right\}$ and $R_{u_{1}}^{i} \geq R_{u_{2}}^{i}$. If a NE channel allocation $\mathbb{X}$ is MMCPNE then the following conditions hold:

- $R_{u_{1}}^{i}-R_{u_{2}}^{i} \leq\left(1 / \delta^{-}-1 / \delta^{+}\right)$and

- case 1: if $R_{u_{1}}^{i} \neq R_{u_{2}}^{i}$ then there does not exist two channels $b \in \mathcal{C}^{+}$and $c \in \mathcal{C}^{-}$such that $k_{u_{1}, b}=k_{u_{2}, b}=1$ whereas $k_{u_{1}, c}=k_{u_{2}, c}=0$,

- case 2: if $R_{u_{1}}^{i}=R_{u_{2}}^{i}$ then there does not exist four channels $\left\{b_{1}, b_{2}\right\} \in \mathcal{C}^{+}$and $\left\{c_{1}, c_{2}\right\} \in \mathcal{C}^{-}$such that $k_{u_{1}, b_{i}}=k_{u_{2}, b_{i}}=1, \forall i$ whereas $k_{u_{1}, c_{j}}=k_{u_{2}, c_{j}}=0, \forall j$.

We could not prove that the set of conditions in Theorem 2 is sufficient to enable a NE channel allocation to be MMCPNE, neither could we find a counterexample, where the conditions hold and the NE channel allocation is not MMCPNE. Hence, we formulate the following conjecture.

\section{CONCLUSION}

In this paper, we have studied the problem of competitive channel allocation among devices that use multiple radios in the multi-hop system. We first analyze that NE and CPNE channel allocation cannot ensure the best performance in terms of end-to-end rate in multi-hop system. Then we propose a new coalition-proof Nash equilibrium, denoted by MMCPNE, to ensure that the multi-hop links are able to achieve high performance as well as the individual players. We investigate the existence of MMCPNE and propose the necessary and sufficient conditions for the existence of MMCPNE. Finally, we provide a two-step algorithm to achieve the MMCPNE state. We study their convergence properties theoretically.

\section{ACKNOWLEDGMENT}

This work is supported by NSF China (No. 60702046); China Ministration of Education (No. 20070248095); Shanghai Jiaotong University Young Faculty Funding; Shanghai Jiaotong University Pre-Research Funding; Qualcom China Research Grant. NSF China \#60672067.

\section{REFERENCES}

[1] J. van den Heuvel, R. A. Leese, and M. A. Shepherd. Graph labeling and radio channel assignment. Journal of Graph Theory, 29:263-283, 1998.

[2] I. Katzela and M. Naghshineh. Channel assignment schemes for cellular mobile telecommunication systems: a comprehensive survey. IEEE Personal Communications, 3(3):10-31, Jun 1996.

[3] Hac A and Z. Chen. Hybrid channel allocation in wireless networks. In Proceedings of the IEEE Conference on Vehicular Technology Conference (VTC 1999), 50(4):2329-2333, Sept. 1999.

[4] A. Mishra, S. Banerjee, and W. Arbaugh. Weighted coloring based channel assignment for WLANs. Mobile Computing and Communications Review (MC2R), 9(3), 2005.

[5] I. F. Akyildiz, X. Wang, and W. Wang. Wireless mesh networks: A survey. Computer Networks, Elsevier North-Holland, Inc., 47:445-487, March 2005.

[6] A. Raniwala and T.-C. Chiueh. Architecture and algorithms for an IEEE 802.11-based multi-channel wireless mesh network. In Proceedings of the IEEE Conference on Computer Communications (INFOCOM '05), March 13-17 2005.

[7] M. Cagalj, S. Ganeriwal, I. Aad, and J.-P. Hubaux. On selfish behavior in CSMA/CA networks. In Proceedings of the IEEE Conference on Computer Communications (INFOCOM '05), March 13-17 2005.

[8] J. Konorski. Multiple access in ad-hoc wireless LANs with noncooperative stations. In Proceedings of Networking 2002, pages 1141-1146, 2002.

[9] A. B. MacKenzie and S. B. Wicker. Stability of multipacket slotted aloha with selfish users and perfect information. In Proceedings of the IEEE Conference on Computer Communications (INFOCOM '03), March 30April 32003.

[10] M. M. Halldorsson, J. Y. Halpern, L. (Erran) Li, and V. S. Mirrokni. On spectrum sharing games. In Proceedings of the 23rd annual ACM symposium on Principles of Distributed Computing (PODC 2004), pages 107-114, July 25-28 2004.

[11] M. Felegyhazi, M. Cagalj, S. S. Bidokhti, and J.-P. Hubaux. Noncooperative Multi-radio Channel Allocation in Wireless Networks. In Proceedings of the IEEE Conference on Computer Communications (INFOCOM '07), March 13-17 2007.

[12] A. Adya, P. Bahl, J. Padhye, A. Wolman and L. Zhou. A multi-radio unification protocol for IEEE 802.11 wireless networks. In Proc. of Broadnets '04, pages 344-354, 2004.

[13] G. Bianchi. Performance analysis of the IEEE 802.11 distributed coordination function. IEEE Journal on Selected Areas in Communication, 18(3), March 2000.

[14] M. Felegyhazi and J.-P. Hubaux. Game theory in wireless networks: A tutorial. Technical Report LCA-REPORT-2006-002, EPFL, Feb. 2006.

[15] Mo Li and Yunhao Liu, "Rendered Path: Range-Free Localization in Anisotropic Sensor Networks with Holes", ACM MobiCom 2007, Montreal, Quebec, Canada, September 2007.

[16] B. D. Bernheim, B. Peleg, and M. D. Whinston. Coalition-proof Nash equilibria: I concepts. Journal of Economic Theory, 42(1), June 1987.

[17] T. S. Rappaport. Wireless Communications: Principles and Practice (2nd Edition). Prentice Hall, 2002.

[18] Mo Li and Yunhao Liu, "Underground Structure Monitoring with Wireless Sensor Networks", ACM/IEEE IPSN, Cambridge, Massachusetts, USA, April, 2007.

[19] M. Schwartz. Mobile Wireless Communications. Cambridge Univ. Press, 2005.

[20] D. Fudenberg and J. Tirole. Game Theory. MIT Press, 1991. 